\title{
A study on the mechanism of Wnt inhibitory factor 1 in osteoarthritis
}

\author{
Zhiyong Zhu, Xizhuang Bai, Huisheng Wang, Xi Li, Guoqiang Sun, Pan Zhang
}

Department of Sports Medicine and Joint Surgery, The People's Hospital of China Medical University, Shenyang, Liaoning, China

Submitted: 1 November 2017

Accepted: 26 November 2017

Arch Med Sci 2020; 16 (4): 898-906

DOI: https://doi.org/10.5114/aoms.2020.95667

Copyright $\odot 2020$ Termedia \& Banach

\section{Abstract}

Introduction: In our study we aimed to investigate the mechanism of Wnt inhibitory factor 1 (WIF1) on regulating chondrocyte proliferation and apoptosis via reactive oxygen species (ROS) and the $W n t / \beta$-catenin signaling pathway in osteoarthritis (OA).

Material and methods: Osteoarthritis chondrocytes were treated with interleukin $1 \beta$ (IL-1 $\beta)$ to simulate an inflammatory condition. Quantitative real-time polymerase chain reaction (qRT-PCR) and western blot were applied for detecting WIF1 expression in OA chondrocytes. MTT assay and flow cytometry were carried out to analyze the cell proliferation and apoptosis. Content of ROS was detected using flow cytometry, and activity of the Wnt/ $\beta$-catenin signaling pathway was detected using immunofluorescence, western blot and luciferase reporter assay. Western blot and enzyme-linked immunosorbent assay (ELISA) were performed to detect the expression of apoptosis-related proteins and secretion of matrix metalloproteinases (MMPs).

Results: WIF1 expression in OA chondrocytes was significantly lower than in normal chondrocytes. After WIF1 CDNA transfection, the aberrantly high ROS level in OA chondrocytes was down-regulated, which led to the increase of proliferation and reduction of apoptosis. The Wnt/ $\beta$-catenin signaling pathway was suppressed by WIF1 overexpression and the secretion of MMPs was therefore reduced.

Conclusions: Up-regulation of WIF1 would promote proliferation and suppress apoptosis of OA chondrocytes through eliminating ROS production and reduce secretion of MMPs via blocking the $W n t / \beta$-catenin signaling pathway.

Key words: osteoarthritis, Wnt inhibitory factor 1 , reactive oxygen species, Wnt/B-catenin.

\section{Introduction}

As a progressively degenerative joint disorder, osteoarthritis (OA) is characterized by extracellular matrix (ECM) molecules' degradation, articular cartilages loss, and osteophyte formation. It would possibly cause chronic disability in the elderly and is one of the major health problems worldwide [1, 2]. Apart from palliative pain control and physiotherapy, no rational medical therapy is currently available for OA treatment before receiving joint replacement therapy [3].

The current views hold that osteoarthritis occurs in all joint organs, such as menisci, subchondral bone, periarticular muscle, and synovium [4]. Many studies have demonstrated the pathological mechanisms of carti-

\author{
Corresponding author: \\ Xizhuang Bai MD \\ Department of Sports \\ Medicine and Joint Surgery \\ The People's Hospital of \\ China Medical University \\ 33 Wenyi Road \\ Shenhe District \\ Shenyang 110016 \\ Liaoning, China \\ Phone: +86024 24016114 \\ E-mail:yxsqilu_1@163.com
}


lage loss [5, 6]. Related studies showed that chondrocyte hypertrophy was connected with damaged calcified cartilage in OA which promoted the degeneration of cartilage [7]. Recent studies have revealed that the progression of $\mathrm{OA}$ is closely related to oxidative stress as well as reactive oxygen species (ROS) [8]. In normal chondrocytes, ROS at moderate levels work as indispensable second messengers and participate in many normal physiological processes. However, in OA pathological situations, excessive ROS trigger the oxidative damage of the cells, oxidizing cellular lipids, proteins, carbohydrates as well as DNAs, and therefore induce cell apoptosis [9]. Furthermore, some researchers claimed that matrix metalloproteinases (MMPs) secreted by OA cells would degrade the cartilage and ECM, whose structure and function were orchestrated by chondrocytes, especially MMP-1, MMP-3 and MMP-13 [10-12]. Catabolic enzymes of chondrocytes achieve tolerance to mechanical stress as well as elasticity of articular cartilages, which is compromised in OA [13]. As for the poor repair and regeneration ability of cartilage, the development of osteoarthritis could be inhibited by enhancing the proliferation of chondrocytes [14]. Therefore, the proliferation ability of chondrocytes is an important factor in delaying the degradation of cartilage, and it could be enhanced via reducing the ROS level as well as MMP secretion.

Since Wnt was first identified by Nusse and Varmus in 1982, the Wnt gene family members have been identified one by one [15]. The Wnt gene family has been evidenced to be involved in cell proliferation, cell migration, a string of complicated metabolic pathways and other activities [16]. Some studies showed that the abnormal expression of the Wnt pathway is connected with different malignant tumors and other diseases, including OA [17]. Wht inhibitory factor-1 (WIF1), as a key antagonist, could bind to Wnt proteins directly in the extracellular space and thus suppress the upstream of Wnt/ $\beta$-catenin signaling pathway [18]. Some studies demonstrated that WIF1 expression was downregulated in many human malignancies such as glioma and astrocytoma [18]. In addition, previous studies showed that WIF1 could impede Wnt3a-mediated inhibition of chondrogenesis [19]. Recent studies showed a possible linkage among ROS, WIF1 and the Wnt/ $\beta$-catenin signaling pathway, which could lead to cell apoptosis [20-22]. Therefore, we supposed that WIF1 may regulate ROS production and the $W n t / \beta$-catenin signaling pathway in chondrocytes.

In our study we aimed to investigate the effect of WIF1 in OA cartilage chondrocytes. We transfected WIF1 cDNA into chondrocytes, and tested the cell proliferation and apoptosis. Wnt/ $\beta$-caten- in signaling pathway and ROS level were explored to assess how WIF1 affected the OA. Since there have been few studies about WIF1 and ROS in osteoarthritis, the results of this study would reveal the connection among WIF1, ROS and chondrocyte viability, which might provide a new therapeutic theory about OA treatment.

\section{Material and methods}

\section{Tissue samples}

Osteoarthritis cartilage tissue samples were collected from 10 OA patients (4 males, 6 females, age 55-78, average age 68.5 years) who received sodium hyaluronate injection. Normal cartilage samples were collected from 10 traumatic knee joint fracture patients without OA ( 5 males, 5 females, age 47-69, average 58.2 years). All the patients received knee arthroplasty in the People's Hospital of China Medical University during the period between December 2015 and October 2016. All the patients have signed the informed consent about this research and all procedures of this research were approved by the Ethics Committee of the People's Hospital of China Medical University.

\section{Cell culture and transfection}

Cartilage tissue fragments were washed with Hank's balanced salt solution (Gibco, Grand Island, NY, USA) three times and then treated with $0.25 \%$ trypsin (Gibco) at $37^{\circ} \mathrm{C}$. Digestion was ended when turbidity appeared in the solution. Dispersive cells were collected and cultured in Dulbecco's modified eagle medium (DMEM, Gibco) containing $10 \%$ fetal bovine serums (FBS, Gibco) and incubated at $37^{\circ} \mathrm{C}$ in humid air with $5 \% \mathrm{CO}_{2}$. After 2 passages, chondrocytes were available for the following experiments.

Osteoarthritis chondrocytes in the logarithmic phase were transfected with WIF1 CDNA plasmid (Vigenebio, Shandong, China) using Lipofectamine 2000 (Invitrogen, Carlsbad, CA, USA) according to the manufacturer's protocol. Human interleukin $1 \beta$ (IL-1 $\beta, 10 \mathrm{ng} / \mathrm{ml}$, Sigma-Aldrich, St. Louis, MO, USA), antioxidant acetylcysteine (NAC, 1/2/5 mM, Sigma-Aldrich) and $\beta$-catenin inhibitor XAV-939 ( $0.5 \mu \mathrm{M}$, Sigma-Aldrich) were used to treat the OA chondrocytes in different groups.

\section{Quantitative real-time polymerase chain reaction (qRT-PCR)}

Total RNA of the OA chondrocytes was extracted using Trizol reagent (Beyotime, Shanghai, China). A DNA Reverse Transcription Kit (Applied Biosystems, CA, USA) was used to perform the reverse transcription, and SYBR Select Master Mix in $A B I$ Prism 7000 Sequence Detection (Applied 
Table I. Primers used for qRT-PCR

\begin{tabular}{|lcc|}
\hline Genes & \multicolumn{1}{c|}{ Forward primer 5' $^{\prime} \mathbf{3}^{\prime}$} & Reverse primer 5' $^{\prime} \mathbf{3}^{\prime}$ \\
\hline WIF1 & TGCCTCACAAGGCATCAGTT & GGAACCCATCAGGACACTCG \\
\hline c-Myc & GGCCCCCAAGGTAGTTATCC & CGTTTCCGCAACAAGTCCTC \\
\hline Cyclin D1 & CAATGACCCCGCACGATTTC & AAGTTGTTGGGGCTCCTCAG \\
\hline GAPDH & CGGAGTCAACGGATTTGGTCGTAT & AGCCTTCTCCATGGTGGTGAAGAC \\
\hline
\end{tabular}

Biosystems) was used to perform the qRT-PCR. GAPDH was used as the internal reference, and the relative expression of mRNAs was calculated using the $2^{-\triangle \Delta C T}$ method. Experiments were repeated in triplicate for accuracy. Primer sequences are shown in Table I.

\section{Western blot}

Osteoarthritis chondrocytes were treated with RIPA lysis buffer (Beyotime), and the concentration of proteins was tested using the Advanced BCA Protein Assay Kit (Beyotime). After SDSPAGE separation, proteins were transferred onto a polyvinylidene difluoride (PVDF) membrane for $1 \mathrm{~h}$ incubation with $1 \%$ bovine serum albumin (BSA, Sigma-Aldrich) and then washed with PBST. Primary antibodies were added and incubated overnight at $4^{\circ} \mathrm{C}$ to bind the target proteins, then secondary antibody was added and incubated for $1 \mathrm{~h}$ at $37^{\circ} \mathrm{C}$. Primary antibodies were rabbit antiWIF1, anti- $\beta$-catenin, anti-lamin $B 1$, anti- $\beta$-actin and anti-GAPDH (1:2000, $1: 1000,1: 1000$, $1: 2000,1: 2000$ respectively, Abcam, Cambridge, MA, USA). Secondary antibody was HRP-labeled goat anti-rabbit IgG (1:2000, Abcam). Blots were filmed by Vilber Lourmat (Marnela Vallée, France) and analyzed by Quantity One software (Bio-Rad, Hercules, CA, USA).

\section{Enzyme-linked immunosorbent assay (ELISA)}

Osteoarthritis chondrocytes were seeded onto 96-well plates and incubated at $37^{\circ} \mathrm{C}$ in humid air with $5 \% \mathrm{CO}_{2}$ for $24 \mathrm{~h}$. Culture supernatant was collected, and then ELISA was performed using a Quantikine ELISA Kit (R\&D Systems, Abingdon, UK) according to the manufacturer's protocol. The OD value was then detected using the microplate reader. Primary antibodies used were rabbit anti-MMP-1, anti-MMP-3 and anti-MMP-13 (all $0.5 \mu \mathrm{g} / \mathrm{ml}$, Abcam), and secondary antibody was HRP-labeled goat anti-rabbit lgG $(0.5 \mu \mathrm{g} / \mathrm{ml}$, Abcam).

\section{MTT assay}

Osteoarthritis chondrocytes with WIF1 siRNA transfected were planted into 96-well plates af- ter digestion by $0.25 \%$ trypsin. After being cultured for $1 / 2 / 3 / 4 / 5$ days, cells in 96-well plates were treated with $20 \mu$ of MTT reagent for $4 \mathrm{~h}$. Then supernatant was removed and $150 \mu \mathrm{l}$ of dimethyl sulfoxide (DMSO) was added to dissolve formazan. The optical density (OD) value of each well was obtained by the microplate reader at the wavelength of $570 \mathrm{~nm}$.

\section{Flow cytometry}

For apoptosis test: After digestion by $0.25 \%$ trypsin (Gibco) for $24 \mathrm{~h}$, the cells were collected into flow tubes and the supernatant was discarded. After washing with PBS three times, $150 \mu \mathrm{l}$ of binding buffer and $1 \mu$ l of Annexin V FITC (SigmaAldrich) were added to each tube, followed by incubation for $15 \mathrm{~min}$ in the dark at room temperature. The cells were then treated with $100 \mu \mathrm{l}$ of propidium iodide (PI, Sigma-Aldrich), and the cell apoptosis was determined by the flow cytometer.

For reactive oxygen species (ROS) level test: The cells were planted into 6-well plates and incubated for $24 \mathrm{~h}$. To each well we added $1 \mathrm{ml}$ of DCFH-DA (Sigma-Aldrich) which was diluted by DMEM (DCFH-DA : DMEM = $1: 1000$ ) for $20 \mathrm{~min}$ culture. After washing with DMEM, cells were collected and we analyzed dichlorofluorescein (DCF) fluorescence intensity, which could reflect ROS level.

\section{Immunofluorescence}

The cells were plated in a 24-well plate and cultured for $24 \mathrm{~h}$, then fixed with $4 \%$ paraformaldehyde for 20 min. Goat serum was used to block the cells for $1 \mathrm{~h}$, and then primary antibody (rabbit anti-human $\beta$-catenin, 1 : 100, Abcam) was added. After overnight incubation, secondary antibody (Alexa Fluor 488-labeled goat anti-rabbit IgG, $1: 200$, Abcam) was added and the cells were incubated in the dark for $1 \mathrm{~h}$. After staining with DAPI (Sigma-Aldrich) for $5 \mathrm{~min}$, the samples were filmed by the fluorescence microscope.

\section{Luciferase reporter assay}

TOP/FOP flash plasmid (Promega, Madison, WI, USA) and WIF1 cDNA plasmid were co-transfected into OA chondrocytes using Lipofectamine 2000. 
After being cultured for $48 \mathrm{~h}$, OA chondrocytes $\left(3 \times 10^{5}\right.$ cells) were planted on the top of a 24-well plate which was coated with Matrigel, and then treated with IL-1 $\beta$ (1 nM) and XAV-939 (0.5 $\mu \mathrm{M})$. Cells were analyzed by the Dual-Luciferase Reporter Assay System (Promega) according to the manufacturer's protocol, and luciferase activity was examined by the luminometer to show the transcriptional activity of $\beta$-catenin.

\section{Statistical analysis}

All the data were analyzed with SPSS 21.0 (IBM, NY, USA) and presented as mean \pm standard deviation (SD). Student's $t$ test and one-way analysis of variance (ANOVA) were used to detect differences between groups. Differences were considered to be statistically significant at $p<0.05$.

\section{Results}

\section{WIF1 was low-expressed in human OA chondrocytes}

Considering that the inflammation of OA chondrocytes collected from cartilage tissues was mitigated after culture in the medium, we treated $\mathrm{OA}$ chondrocytes with IL-1 $\beta$ to simulate the inflammatory condition. We then detected the differential expressed gene WIF1 in normal and OA chondrocytes. Both qRT-PCR and western blot showed that WIF1 expression of human OA chondrocytes was significantly lower than that in normal chon- drocytes $(p<0.01)$, while that of IL-1 $\beta$ treated OA chondrocytes was lower than OA chondrocytes $(p<0.05)$, indicating that WIF1 was negatively related to chondrocyte inflammation (Figures $1 \mathrm{~A}, \mathrm{~B}$ ).

\section{WIF1 down-regulated ROS level in OA chondrocytes}

The DCF fluorescence intensity of OA chondrocytes decreased significantly compared with normal chondrocytes, while that of IL-1 $\beta$ treated OA chondrocytes was even higher than OA chondrocytes, indicating that the ROS level was up-regulated in OA $(p<0.01$, Figure $2 A)$. Then IL- $1 \beta$ treated OA chondrocytes were incubated with NAC (1/2/5 mM) for $12 \mathrm{~h}$, and ROS level was gradually decreased with the increase of NAC concentration, showing the efficiency of NAC in ROS elimination $(p<0.001$, Figure 2 B). After WIF1 cDNA transfection, ROS level of IL-1 $\beta$ treated OA chondrocytes was significantly decreased ( $p<0.01$, Figure $2 \mathrm{C}$ ), revealing that WIF1 could suppress ROS production.

\section{WIF1 promoted proliferation and reduced apoptosis of OA chondrocytes through suppressing ROS production}

We transfected OA chondrocytes with WIF1 CDNA and observed the results of qRT-PCR, which demonstrated that WIF1 cDNA could remarkably enhance the expression of WIF1 $(p<0.01$, Figure $3 \mathrm{~A}$ ), suggesting that we succeeded in over-
A

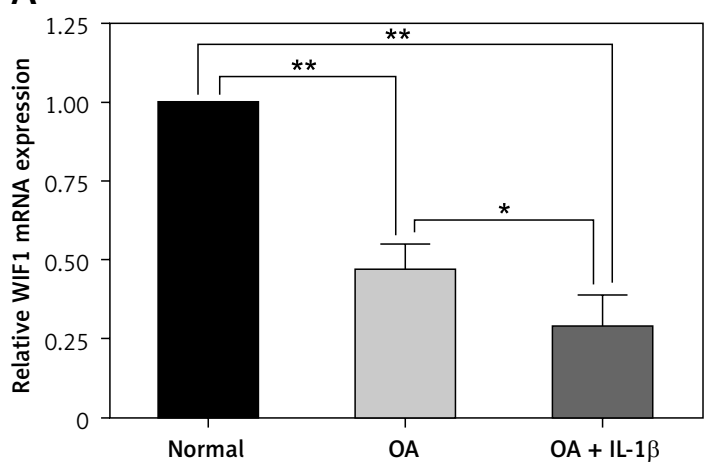

B
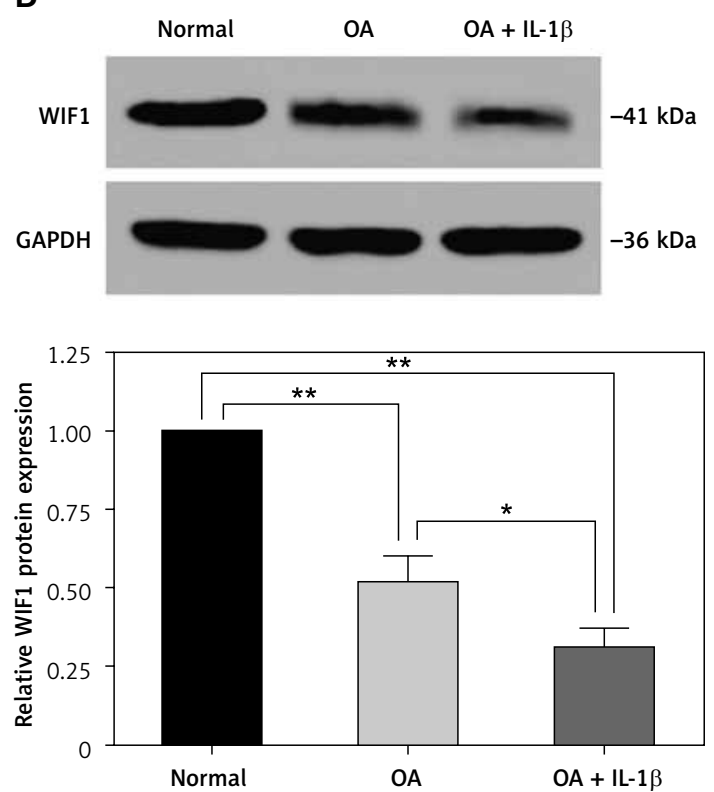

Figure 1. WIF1 was low-expressed in osteoarthritis (OA) chondrocytes. A - qRT-PCR: WIF1 expression of OA chondrocytes was significantly lower compared with normal chondrocytes, while that of IL-1 $\beta$ treated OA chondrocytes was lower than non-treated OA chondrocytes. B - Western blot: WIF1 expression of OA chondrocytes was significantly lower compared with normal chondrocytes, while that of IL-1 $\beta$ treated OA chondrocytes was lower than non-treated OA chondrocytes $\left({ }^{\star} p<0.05,{ }^{* *} p<0.01\right)$ 
A

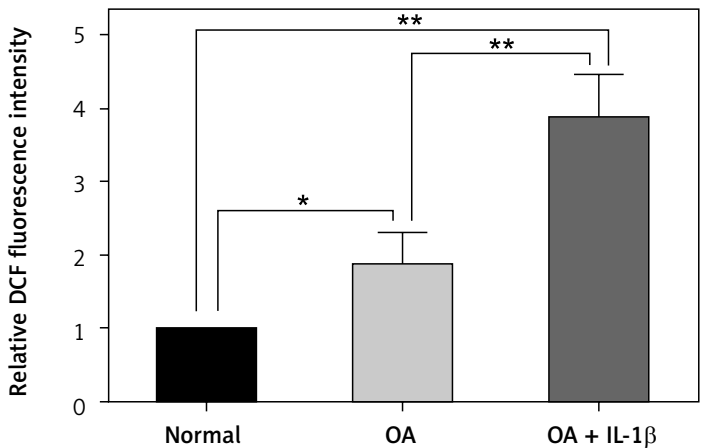

C

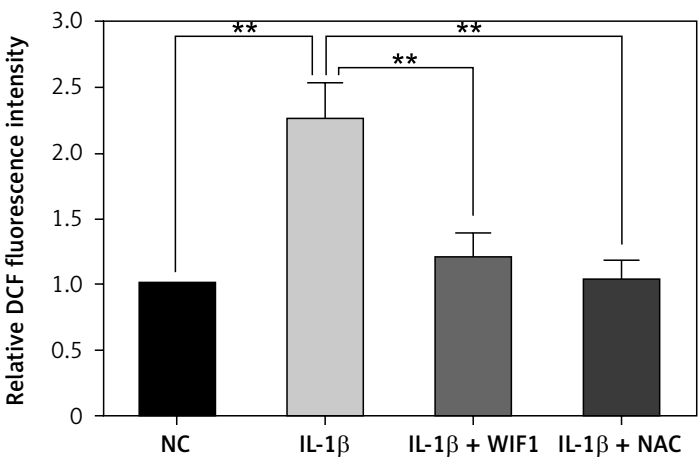

expressing WIF1. Cellular experiments were then performed to test the effect of up-regulated WIF1. MTT assay revealed that both up-regulated WIF1 and NAC ( $5 \mathrm{mM})$ would promote the proliferation of IL- $1 \beta$ treated OA chondrocytes $(p<0.01$, Figure $3 \mathrm{~B}$ ); according to flow cytometry results, the apoptosis rate of IL- $1 \beta$ treated OA chondrocytes was significantly reduced by WIF 1 and NAC $(p<0.01$, Figure $3 \mathrm{C}$ ). Western blot was performed to detect apoptosis-related proteins caspase-3, PARP, Bax and $\mathrm{Bcl}-2$. The expression of cleaved caspase-3, cleaved PARP and Bax was significantly reduced, while expression of anti-apoptotic protein $\mathrm{BCl}-2$ was increased after WIF1 CDNA transfection or NAC treatment, indicating that WIF1 or NAC could suppress cell apoptosis of OA chondrocytes ( $p<$ 0.01 , Figure $3 \mathrm{D}$ ). All these results revealed that WIF1 would protect the viability of chondrocytes via reducing ROS production.

\section{WIF1 blocked Wnt/ $\beta$-catenin signaling pathway and therefore reduced MMP secretion}

Activity of $\beta$-catenin was detected by western blot, immunofluorescence and luciferase reporter assay. Western blot results revealed enhanced nuclear and total $\beta$-catenin expression as well as decreased cytoplasmic $\beta$-catenin expression in IL-1 $\beta$ treated OA chondrocytes compared with OA chondrocytes (negative control), while overexpressing WIF1 or the presence of $\beta$-catenin inhib-
B

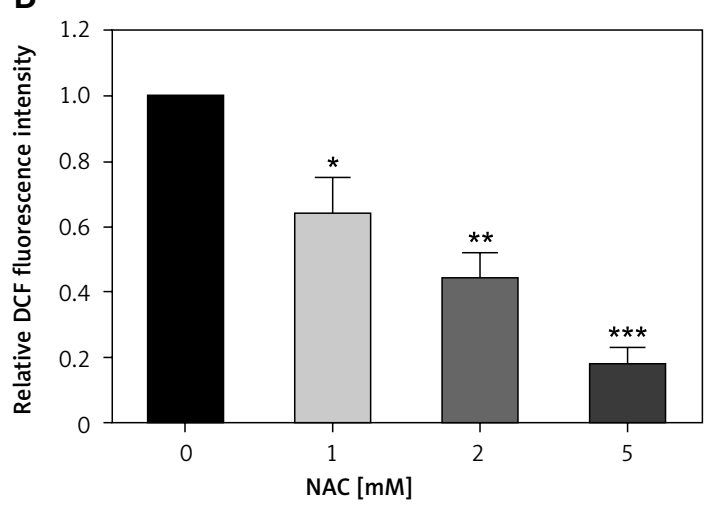

Figure 2. WIF1 was negatively correlated with ROS in osteoarthritis (OA) chondrocytes. A - Flow cytometry: DCF fluorescence intensity of OA chondrocytes was significantly higher than normal chondrocytes, while that of IL- $1 \beta$ treated OA chondrocytes was higher than non-treated OA chondrocytes. B - Flow cytometry: DCF fluorescence intensity of IL-1 $\beta$ treated OA chondrocytes was gradually decreased with the presence of NAC $(1 / 2 / 5 \mathrm{mM})$. $C$ - Flow cytometry: DCF fluorescence intensity of IL-1 $\beta$ treated OA chondrocytes was decreased after WIF1 cDNA transfection or NAC treatment $\left({ }^{\star} p<0.05,{ }^{\star \star} p<0.01,{ }^{\star \star *} p<0.001\right)$

itor XAV-939 showed an inverse result $(p<0.01$, Figure 4 A). Immunofluorescence demonstrated the parallel result that the fluorescence intensity of $\beta$-catenin in the IL- $1 \beta+$ WIF1 and IL-1 $\beta+$ XAV939 group was distinctly lower compared with the IL-1 $\beta$ group ( $p<0.05$, Figure 4 B). Luciferase reporter assay confirmed that the transcriptional activity of $\beta$-catenin, which was enhanced by IL-1 $\beta$ stimulation, could be significantly reduced by WIF1 or XAV-939 $(p<0.01$, Figure 4 C). Moreover, we detected the secretion of MMP-1, MMP-3 and MMP-13 in the cell culture supernatant. All of them were remarkably suppressed by WIF1 and XAV-939 $(p<0.01$, Figure 4 D). All these results confirmed that up-regulated WIF1 blocked the Wnt/ $\beta$-catenin pathway.

\section{Discussion}

Our study focused on the effect of WIF1 in OA. WIF1 expression in OA cartilage tissues was lower than in normal tissues. After WIF1 CDNA transfection, the aberrantly high ROS level declined, and therefore the apoptosis rate of OA chondrocytes decreased while proliferation was significantly enhanced. Up-regulating WIF1 could also block the Wnt/ $\beta$-catenin signaling pathway and reduce the secretion of MMPs.

$\mathrm{OA}$, though widespread, is hard to cure and current therapies only focus on reducing pain and strengthening joint function [4]. Recent studies have shown that many genes are associated 
A

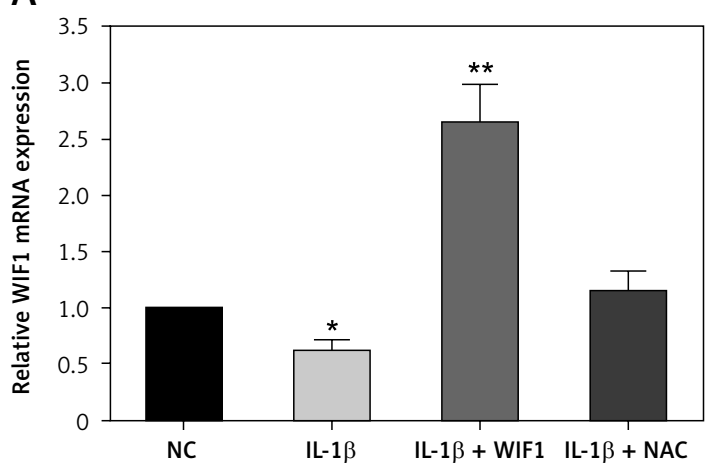

B

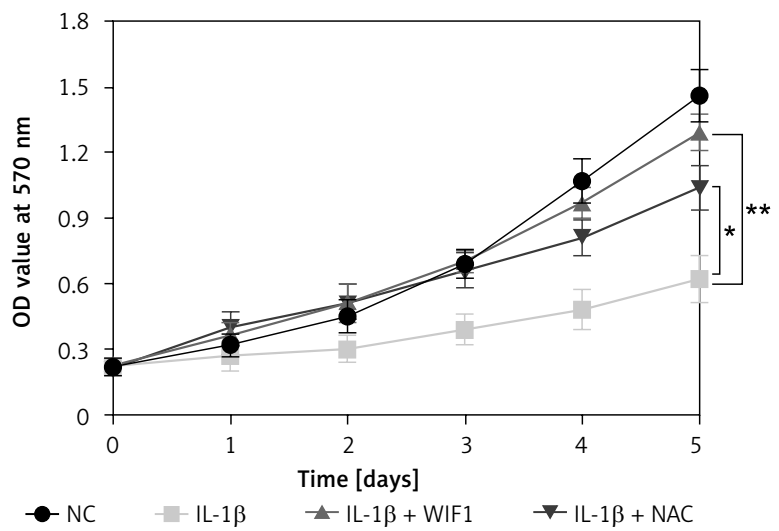

$\rightarrow \mathrm{NC} \quad-\mathrm{IL}-1 \beta \quad \mathrm{IL}-1 \beta+$ WIF1 $\rightarrow$ IL- $1 \beta+\mathrm{NAC}$

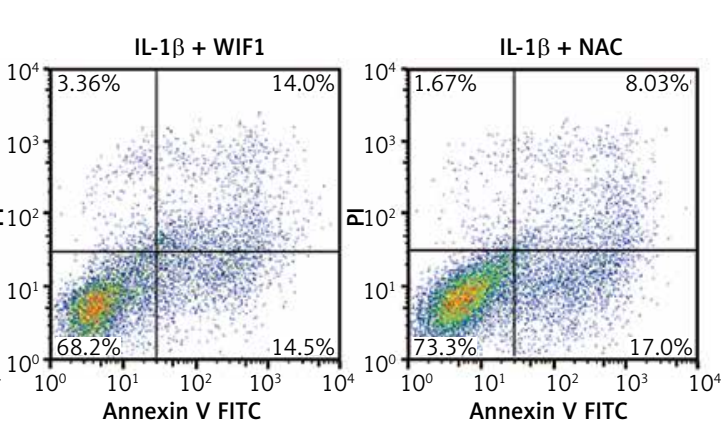

$c$
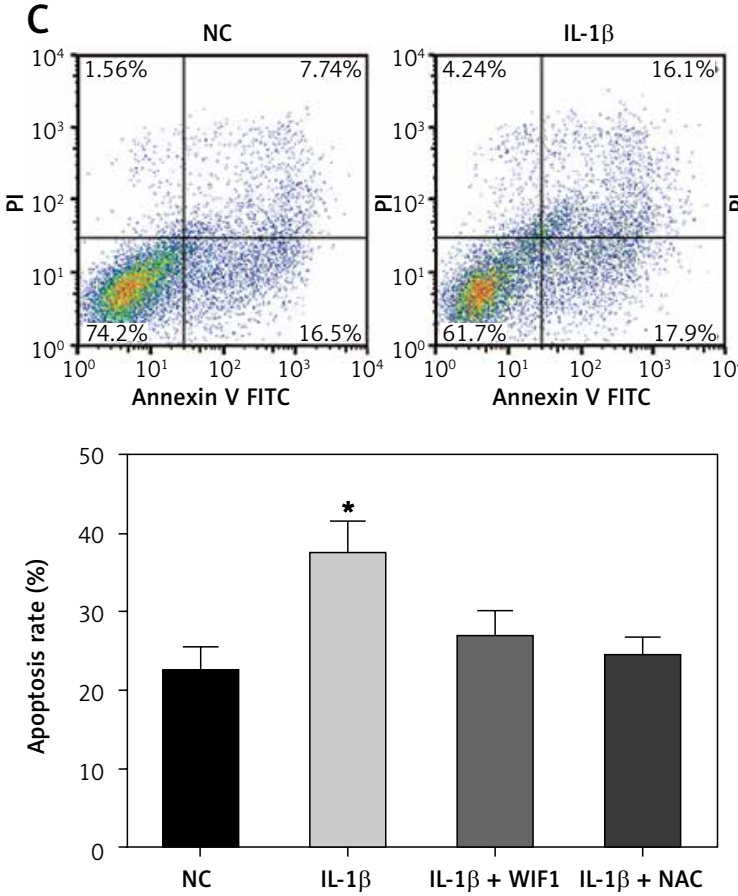

D

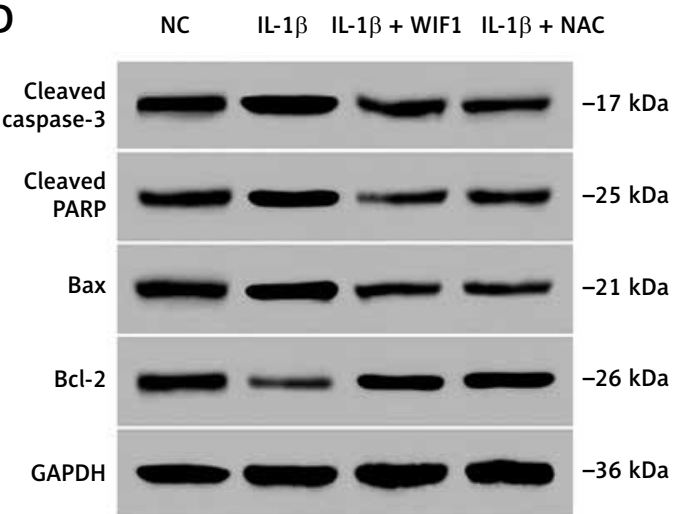

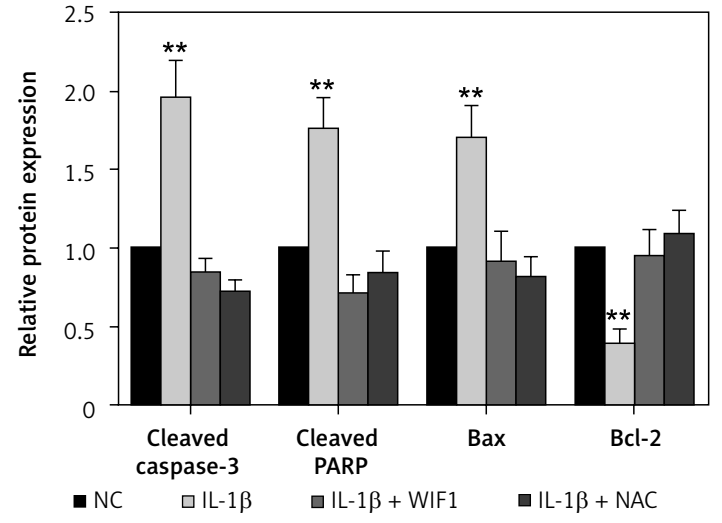

Figure 3. WIF1 promoted proliferation and suppressed apoptosis of osteoarthritis (OA) chondrocytes via down-regulating ROS level in OA chondrocytes. A - qRT-PCR: WIF1 mRNA expression was significantly increased after WIF1 CDNA transfection. B - MTT assay: viability of OA chondrocytes was significantly increased after WIF1 CDNA transfection or NAC $(5 \mu \mathrm{M})$ treatment. $\mathrm{C}$ - Flow cytometry: apoptosis rate of OA chondrocytes was significantly reduced after WIF1 CDNA transfection or NAC treatment. D - Western blot: expression of pro-apoptotic proteins cleaved caspase-3, cleaved PARP and Bax was significantly reduced, while expression of anti-apoptotic protein Bcl-2 was increased after WIF1 CDNA transfection or NAC treatment $\left({ }^{*} p<0.05,{ }^{* *} p<0.01\right.$, compared with negative control group) 
A
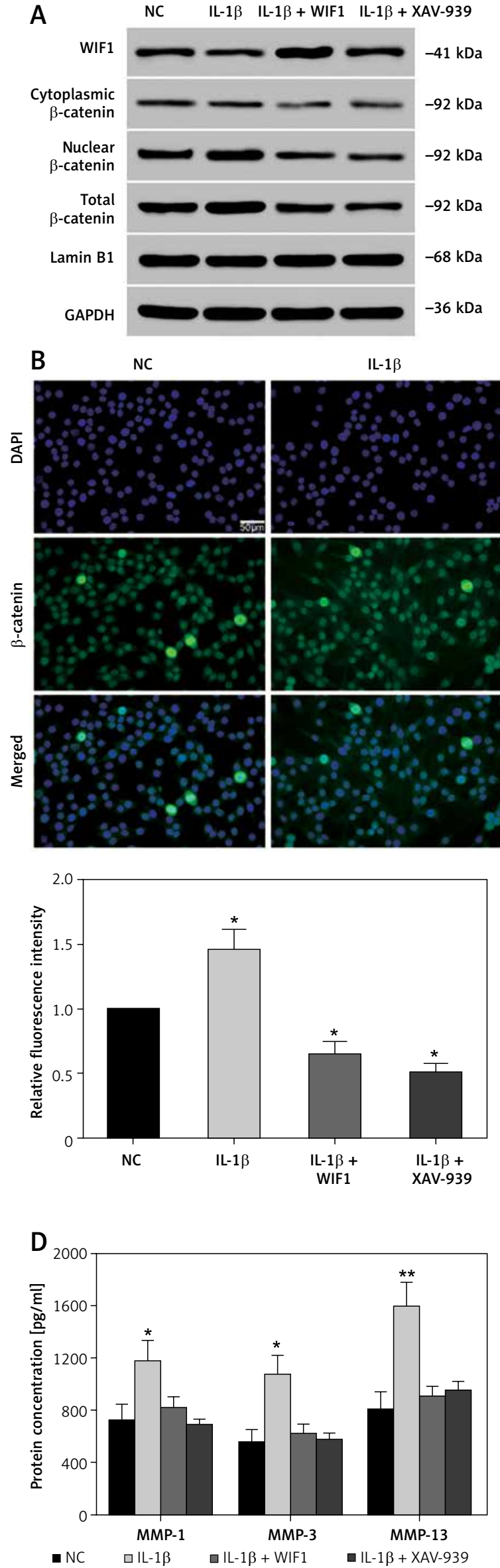

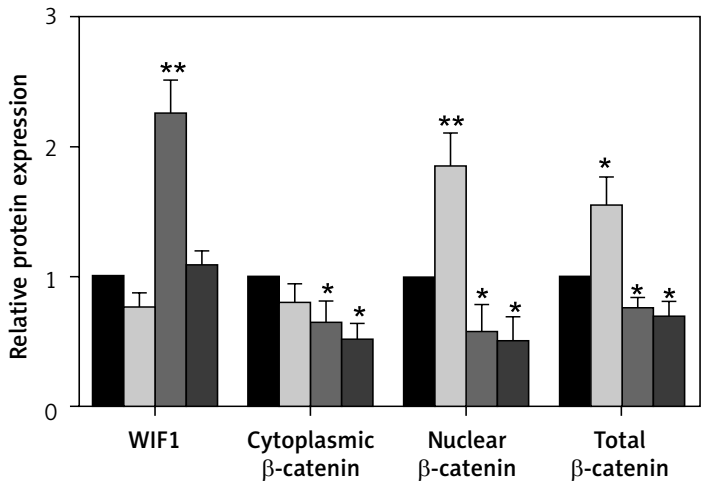

-NC $\quad \mathrm{NIL}-1 \beta \quad \square \mathrm{IL}-1 \beta+$ WIF1 $\quad$ IL-1 $\beta+$ XAV-939 IL-1 $\beta+$ WIF1

$\mathrm{IL}-1 \beta+\mathrm{XAV}-939$

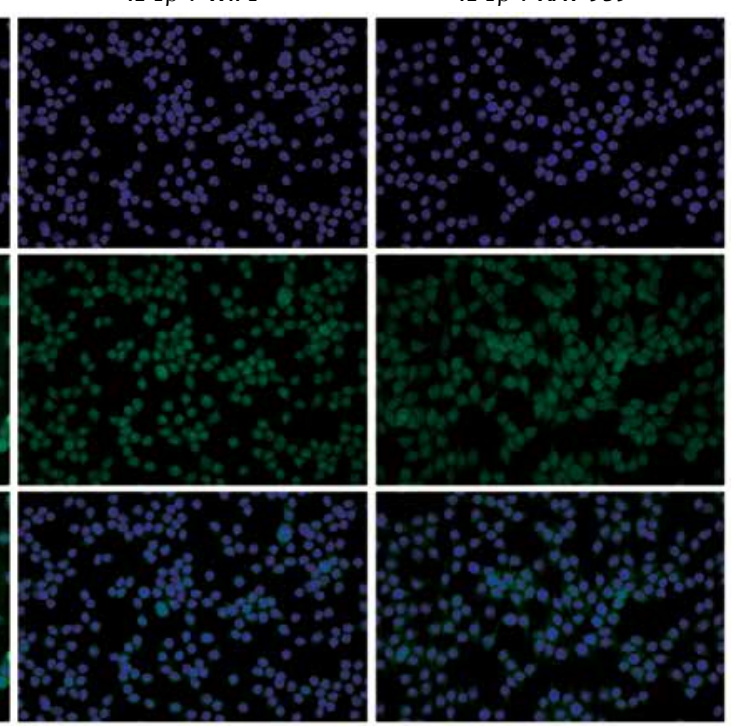

C

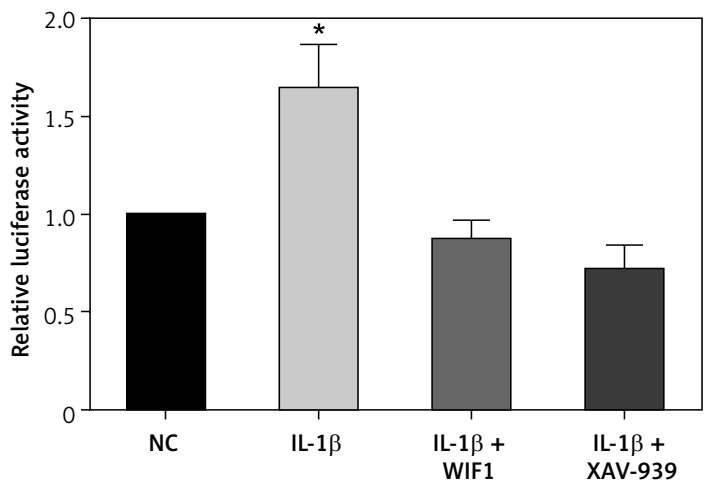

Figure 4. WIF1 blocked Wnt/ $\beta$-catenin pathway and reduced MMP secretion in osteoarthritis (OA) chondrocytes. A - Western blot: WIF1 expression was increased after WIF1 cDNA transfection; cytoplasmic $\beta$-catenin expression was decreased whereas nuclear and total $\beta$-catenin expression was increased after IL-1 $\beta$ stimulation, while cytoplasmic, nuclear and total $\beta$-catenin expressions were all reduced by WIF1 or XAV-939. B - Immunofluorescence: $\beta$-catenin fluorescence intensity, which could be enhanced by IL-1 $\beta$, was significantly reduced by WIF1 or XAV-939. C - Luciferase reporter assay: relative luciferase activity, which could be activated by IL-1 $\beta$, was significantly reduced by WIF1 or XAV-939. D - ELISA: secretion of MMP-1, MMP-3 and MMP-13 of IL-1 $\beta$ treated OA chondrocytes in culture supernatant was significantly decreased after overexpressing WIF1 or being treated with XAV-939 $\left({ }^{*} p<0.05\right.$, ${ }^{* *} p<0.01$, compared with negative control group) 
with osteoarthritis [23]. For example, related studies have shown that the expression levels of tumor necrosis factor $\alpha$ (TNF- $\alpha$ ), osteopontin and nuclear factor $\kappa \mathrm{B}(\mathrm{NF}-\kappa \mathrm{B})$ in patients with knee OA were higher than in healthy controls $[24,25]$. Another study showed that the expression level of SIRT1 was decreased in the OA group, and SIRT1 might act as an indicator for determining formation and development of knee OA [26]. In this study, a lower WIF1 expression in OA cartilage tissues than in normal tissues was displayed. We supposed that WIF1 might have effects on the chondrocytes in OA. We tested the effects of WIF1 by transfecting WIF1 cDNA in chondrocytes, and the results indicated that high WIF1 expression would promote the OA chondrocyte proliferation and reduce the cell apoptosis. Based on this result, we performed our study on its mechanism including the relationship among WIF1, ROS and the Wnt/ $\beta$-catenin pathway.

In the past decades, multi-factorial etiology of OA has been widely discussed, including ROS overproduction and oxidative stress [9]. However, the relationship between ROS and WIF1 was not so compelling. A previous study has evidenced that WIF1 was a ROS marker in systemic sclerosis [20], but whether WIF1 could affect the content of ROS in OA has not been investigated before. Our study demonstrated that WIF1 expression was negatively associated with ROS level in OA, partly illuminating the mechanism of WIF1 in protecting chondrocyte viability.

The connection between WIF1 and the Wnt/ $\beta$-catenin signaling pathway has been widely explored and reported [27]. It was demonstrated that inhibiting WIF1 induced glioma cell growth through the Wnt/ $\beta$-catenin pathway [28]. In this study, we explored how WIF1 affects OA development through the Wnt/ $\beta$-catenin signaling pathway. A recent study showed us the possible relationship. For example, verapamil could prevent the degradation of cartilage in OA by downregulating protein expression in the Wnt/ $\beta$-catenin signaling pathway [3]. Tetrandrine has been evidenced to have potential for the treatment of OA through the Wnt/ $\beta$-catenin signaling pathway [29]. Inhibiting $\mathrm{EZH} 2$ expression can ameliorate the development of osteoarthritis through the Wnt/ $\beta$-catenin pathway [30]. Our study demonstrated that WIF1 could inhibit $\beta$-catenin to transfer from cytoplasm to the nucleus and reduce its transcriptional activity. Moreover, WIF1 could suppress the expression and secretion of MMPs, which were located downstream of $\beta$-catenin. Thus, we confirmed that WIF1 could protect the viability of chondrocytes via blocking the Wnt/ $\beta$-catenin pathway and therefore suppress MMP secretion, reducing aberrant degradation of ECM.
In conclusion, WIF1 promoted OA chondrocyte proliferation and reduced cell apoptosis. The ROS level was reduced while the Wnt/ $\beta$-catenin pathway was activated by overexpressing WIF1. However, the specific molecular mechanism of their interaction was not explored in our study due to some restrictions. In our further studies, more experiments will be performed to analyze the relationships among OA, WIF1, the Wnt/ $\beta$-catenin pathway and ROS, and their actual effects on OA will be tested by in vivo experiments. We supposed that WIF1 could become a potential target to cure osteoarthritis.

\section{Conflict of interest}

The authors declare no conflict of interest.

\section{References}

1. Arden NK, Leyland KM. Osteoarthritis year 2013 in review: clinical. Osteoarthritis Cartilage 2013; 21: 1409-13.

2. Aman A, Nguyen M, Piotrowski T. Wnt/beta-catenin dependent cell proliferation underlies segmented lateral line morphogenesis. Dev Biol 2011; 349: 470-82.

3. Takamatsu A, Ohkawara B, Ito $M$, et al. Verapamil protects against cartilage degradation in osteoarthritis by inhibiting Wnt/beta-catenin signaling. PLoS One 2014; 9: e92699.

4. Hunter DJ. Osteoarthritis. Rheum Dis Clin North Am 2013; 39: xv-xviii.

5. Funck-Brentano T, Lin H, Hay E, et al. Targeting bone alleviates osteoarthritis in osteopenic mice and modulates cartilage catabolism. PLoS One 2012; 7: e33543.

6. Suri S, Walsh DA. Osteochondral alterations in osteoarthritis. Bone 2012; 51: 204-11.

7. van der Kraan PM, van den Berg WB. Chondrocyte hypertrophy and osteoarthritis: role in initiation and progression of cartilage degeneration? Osteoarthritis Cartilage 2012; 20: 223-32.

8. Li D, Xie G, Wang W. Reactive oxygen species: the 2-edged sword of osteoarthritis. Am J Med Sci 2012; 344: 486-90.

9. Lepetsos P, Papavassiliou AG. ROS/oxidative stress signaling in osteoarthritis. Biochim Biophys Acta 2016; 1862: 576-91.

10. Cao K, Wei L, Zhang Z, et al. Decreased histone deacetylase 4 is associated with human osteoarthritis cartilage degeneration by releasing histone deacetylase 4 inhibition of runt-related transcription factor- 2 and increasing osteoarthritis-related genes: a novel mechanism of human osteoarthritis cartilage degeneration. Arthritis Res Ther 2014; 16: 491.

11. Siebelt M, Waarsing JH, Groen HC, et al. Inhibited osteoclastic bone resorption through alendronate treatment in rats reduces severe osteoarthritis progression. Bone 2014; 66: 163-70.

12. Schroeppel JP, Crist JD, Anderson HC, Wang J. Molecular regulation of articular chondrocyte function and its significance in osteoarthritis. Histol Histopathol 2011; 26: 377-94.

13. Li X, Chen J, Liang W, et al. Bushen Zhuangjin Decoction promotes chondrocyte proliferation by stimulating cell cycle progression. Exp Ther Med 2015; 9: 839-44. 
14. Ciorba A, Martini A. Tissue engineering and cartilage regeneration for auricular reconstruction. Int J Pediatr Otorhinolaryngol 2006; 70: 1507-15.

15. Huang Y, Du Q, Wu W, She F, Chen Y. Rescued expression of WIF-1 in gallbladder cancer inhibits tumor growth and induces tumor cell apoptosis with altered expression of proteins. Mol Med Rep 2016; 14: 2573-81.

16. Haase G, Gavert N, Brabletz T, Ben-Ze'ev A. The Wnt target gene L1 in colon cancer invasion and metastasis. Cancers (Basel) 2016; 8. pii: E48.

17. Karamboulas C, Ailles L. Developmental signaling pathways in cancer stem cells of solid tumors. Biochim Biophys Acta 2013; 1830: 2481-95.

18. Malinauskas T, Aricescu AR, Lu W, Siebold C, Jones EY Modular mechanism of Wnt signaling inhibition by Wnt inhibitory factor 1. Nat Struct Mol Biol 2011; 18: 886-93.

19. Surmann-Schmitt C, Widmann N, Dietz U, et al. Wif-1 is expressed at cartilage-mesenchyme interfaces and impedes Wnt3a-mediated inhibition of chondrogenesis. J Cell Sci 2009; 122: 3627-37.

20. Svegliati S, Marrone G, Pezone A, et al. Oxidative DNA damage induces the ATM-mediated transcriptional suppression of the Wnt inhibitor WIF-1 in systemic sclerosis and fibrosis. Sci Signal 2014; 7: ra84.

21. Rharass T, Lemcke H, Lantow M, Kuznetsov SA, Weiss DG, Panakova D. Ca2+-mediated mitochondrial reactive oxygen species metabolism augments Wnt/beta-catenin pathway activation to facilitate cell differentiation. J Biol Chem 2014; 289: 27937-51.

22. Zhang DY, Pan Y, Zhang C, et al. Wnt/beta-catenin signaling induces the aging of mesenchymal stem cells through promoting the ROS production. Mol Cell Biochem 2013; 374: 13-20.

23. Ratnayake M, Tselepi M, Bloxham R, Ploger F, Reynard LN, Loughlin J. A consistent and potentially exploitable response during chondrogenesis of mesenchymal stem cells from osteoarthritis patients to the protein encoded by the susceptibility gene GDF5. PLOS One 2017; 12: e0176523.

24. Qin LF, Wang WC, Fang H, et al. Expression of NF-kappaB and osteopontin of synovial fluid of patients with knee osteoarthritis. Asian Pac J Trop Med 2013; 6: 379-82.

25. Munoz-Valle JF, Oregon-Romero E, Rangel-Villalobos $\mathrm{H}$, et al. High expression of TNF alpha is associated with -308 and -238 TNF alpha polymorphisms in knee osteoarthritis. Clin Exp Med 2014; 14: 61-7.

26. Li Y, Xiao W, Wu P, et al. The expression of SIRT1 in articular cartilage of patients with knee osteoarthritis and its correlation with disease severity. J Orthop Surg Res 2016; 11: 144

27. Xie D, Xie J, Wan Y, et al. Norcantharidin blocks Wnt/ beta-catenin signaling via promoter demethylation of WIF-1 in glioma. Oncol Rep 2016; 35: 2191-7.

28. Guo $M$, Zhang X, Wang G, et al. miR-603 promotes glioma cell growth via Wnt/beta-catenin pathway by inhibiting WIF1 and CTNNBIP1. Cancer Lett 2015; 360: 76-86.

29. Zhou X, Li W, Jiang L, et al. Tetrandrine inhibits the Wnt/ beta-catenin signalling pathway and alleviates osteoarthritis: an in vitro and in vivo study. Evid Based Complement Alternat Med 2013; 2013: 809579.

30. Chen L, Wu Y, Wu Y, Wang Y, Sun L, Li F. The inhibition of EZH2 ameliorates osteoarthritis development through the Wnt/beta-catenin pathway. Sci Rep 2016; 6: 29176. 